\title{
Catheter ablation or surgical therapy in moderate-severe tricuspid regurgitation caused by long-standing persistent atrial fibrillation. Propensity score analysis
}

Jiangang Wang ${ }^{1 * \dagger} \mathbb{O}$, Songnan $\mathrm{Li}^{2 \dagger}$, Qing Ye ${ }^{1 \dagger}$, Xiaolong Ma', Yichen Zhao ${ }^{1}$, Jie Han ${ }^{1}$, Yan $\mathrm{Li}^{1}$, Shuai Zheng ${ }^{1}$, Kemin Liu', Meng He', Wen Yu', Junhui Sun ${ }^{1}$ and Xu Meng ${ }^{1}$

\begin{abstract}
Background: This study aimed to describe the mid-term outcomes of different treatments in patients with atrial fibrillation caused tricuspid regurgitation.

Methods: A retrospective study of patients diagnosed as atrial fibrillation caused moderate-severe tricuspid regurgitation undergoing ablation $(n=411)$ were reviewed. The surgical cohort $(n=114)$ underwent surgical ablation and tricuspid valve repair; the catheter cohort $(n=279)$ was selected from those patients who had catheter ablation.

Results: The estimated actuarial 5-year survival rates were 96.8\% (95\% Cl: 92.95-97.78) and 92.0\% (95\% Cl: 85.26-95.78) in the catheter and surgical cohort, respectively. Tethering height was showed as independent risk factors for recurrent atrial fibrillation and tricuspid regurgitation in both cohorts. A matched group analysis using propensity-matched was conducted after categorizing total patients by tethering height $<6 \mathrm{~mm}$ and $\geq 6 \mathrm{~mm}$. Kaplan-Meier analysis showed in patients with tethering height $<6 \mathrm{~mm}$, there were no differences in survival from mortality, stroke, recurrent atrial fibrillation and tricuspid regurgitation between two groups. In patients with tethering height $\geq 6 \mathrm{~mm}$, there were significantly higher cumulative incidence of stroke $(95 \% \mathrm{Cl}, 0.047-0.849 ; P=0.029)$, recurrent atrial fibrillation $(95 \% \mathrm{Cl}$, $0.357-09738 ; P=0.039)$ and tricuspid regurgitation $(95 \% \mathrm{Cl}, 0.359-0.981 ; P=0.042)$ in catheter group.

Conclusions: Atrial fibrillation caused tricuspid regurgitation resulted in less leaflets coaptation, which risk the recurrence of atrial fibrillation and tricuspid regurgitation. Patients whose tethering height was less than $6 \mathrm{~mm}$ showed satisfying improvement in tricuspid regurgitation with the restoration of sinus rhythm after catheter ablation. However, in patients with severe leaflets tethering, the results favored surgical over catheter.
\end{abstract}

Keywords: Tricuspid regurgitation, Atrial fibrillation, Outcomes

\footnotetext{
*Correspondence: jiangangwang@ccmu.edu.cn

†Jiangang Wang, Songnan Li and Qing Ye contributed equally to this work. 'Department of Cardiac Surgery, Beijing Anzhen Hospital, Capital Medical University, No.2 Anzhen Road, Chaoyang District, Beijing 100029, PR China Full list of author information is available at the end of the article
}

(c) The Author(s). 2020 Open Access This article is licensed under a Creative Commons Attribution 4.0 International License, which permits use, sharing, adaptation, distribution and reproduction in any medium or format, as long as you give appropriate credit to the original author(s) and the source, provide a link to the Creative Commons licence, and indicate if changes were made. The images or other third party material in this article are included in the article's Creative Commons licence, unless indicated otherwise in a credit line to the material. If material is not included in the article's Creative Commons licence and your intended use is not permitted by statutory regulation or exceeds the permitted use, you will need to obtain permission directly from the copyright holder. To view a copy of this licence, visit http://creativecommons.org/licenses/by/4.0/ The Creative Commons Public Domain Dedication waiver (http://creativecommons.org/publicdomain/zero/1.0/) applies to the data made available in this article, unless otherwise stated in a credit line to the data. 


\section{Introduction}

Functional tricuspid regurgitation (TR) with a normal structural tricuspid valve (TV) leaflets and chordae often occur secondary to long-standing persistent atrial fibrillation (LSPAF) [1, 2]. This causes abnormalities of surrounding or supporting structures, such as annular dilatation and right ventricular (RV) dysfunction, promoting dilation of atrioventricular valve annuli that ultimately results in moderate-severe TR AF (TRAF) [3]. Moreover, increased mortality among patients with TRAF, regardless of only class 1 indication for TV repair during concomitant left heart surgery (Level of Evidence: $\mathrm{C}$ ), has raised interest in various therapeutic strategies [4].

Catheter ablation targeting pulmonary veins (PVs) isolation has become the common treatment for atrial fibrillation (AF). To prevent recurrences in patients with LSPAF, isolation of PVs alone may not be sufficient. A fixed approach is developed for catheter ablation of LSPAF, which consists of bilateral circumferential pulmonary vein antrum isolation (PVAI) ('2C') and three linear ablation sets ('3 L') [5]. Functional atrioventricular valvular regurgitation caused by AF could eliminate by catheter ablation, and the negative atrial remodeling induced by valvular regurgitation could also be reversed, decreasing the recurrence of AF after ablation [6, 7]. However, it is not known whether the AF-caused moderate-severe TR could eliminate after '2C3L' procedure.

Moreover, the development of open surgical ablation of AF has led to their widespread use during cardiac operations [8-11]. Surgical ablation concomitant with TV repair, therefore, might be the optimal treatment for the patients with AF-caused moderate-severe TR. However, the progression of TR and rhythm status after surgical treatment has not been comprehensively evaluated.

Therefore, we have conducted a retrospective cohort study to evaluate the differences between the catheter and surgical treatment in patients with LSPAF caused moderate-severe TR in the mid-term follow-up. Moreover, by assessing the heart rhythm status, and TV and RV functions before and after different treatments, the contributions of these treatments to AF and TR were detected.

\section{Methods}

\section{Patient selection}

The institutional review board at the Beijing Anzhen Hospital, Capital Medical University has approved the study. All patients referred for catheter or surgical ablation for LSPAF with moderate-severe TR between January 2008 and December 2013 were considered eligible for inclusion. Patients underwent surgical ablation concomitant with TV repair were included in the surgical cohort. The catheter cohort included patients who underwent '2C3L' ablation. The exclusion criteria were: 1 ) previous percutaneous coronary intervention or cardiac surgery; 2) patients with more than moderate mitral regurgitation; 3) patients with an ejection fraction of $<40 \%$.

\section{Catheter ablation and surgical procedure}

A fixed approach for ablation of long-standing persistent AF was developed, which consists of bilateral circumferential PVAI ('2C') and three linear ablation sets ('3 L') (Fig. 1). Linear ablation is empirically applied across the mitral isthmus

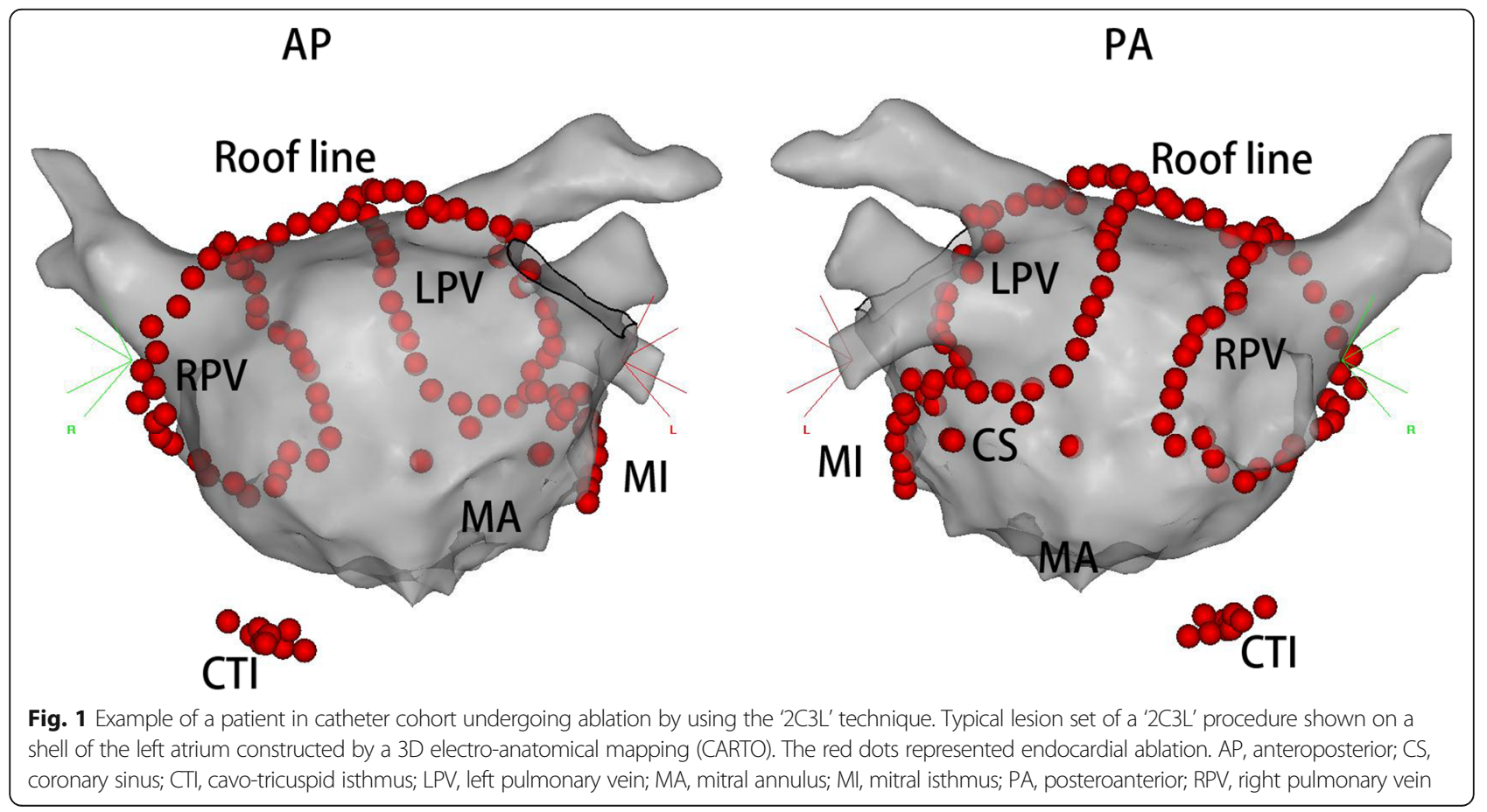


(MI), the left atrial (LA) roof, and the cavo-tricuspid isthmus (CTI). Cardioversion was performed if AF persisted or converted into an organized atrial tachyarrhythmia (OAT) following the initial pulmonary vein (PV) antrum and linear ablations. If cardioversion failed or atrial fibrillation (AF) has immediately relapsed, amiodarone was intravenously administered before repeat cardioversion. After the restoration of sinus rhythm (SR), radiofrequency applications were applied, and if needed, to close the gaps along with the circumferential lesions to achieve pulmonary vein antrum isolation (PVAI) was later verified by a circular mapping catheter. Similarly, conduction across the LA roofline, the MI line, and the CTI line were evaluated by using differential pacing manoeuvers. Also if necessary, the ridge between the LA appendage (LAA) and the left superior PV was targeted. Further ablations were conducted to achieve complete linear block as previously reported. If an OAT occurred after PVAI and documented the blockage of the lines, this tachycardia was then ablated with guidance of 3D electroanatomic mapping and conventional electrophysiological manoeuvres. If AF relapsed after PVAI and linear block across the LA roof, $\mathrm{MI}$ and CTI lines were achieved, then the superior vena cava (SVC) was isolated following cardioversion. Procedural endpoint included the isolation of PV antrum and complete conduction of block across the three ablation lines.

Patients who experienced arrhythmia recurrence after the blanking period were treated with cardioversion, medication, or repeated ablation at the patient's and physician's discretion. A redo procedure followed the '2C3L' strategy where the conduction gaps were targeted, and clinical OATs were also mapped and ablated; however, complex fractionated atrial electrograms (CFAEs) ablation were not performed during the repeat procedure. After completing the PVAI and blockage across the three lines (LA roof, MI, and CTI), burst pacing at $200 \mathrm{~ms}$ was applied from proximal coronary sinus (CS) to induce tachycardia. Only the traceable OAT further underwent ablation, and the endpoint included was that the targeting OAT became non-inducible. Otherwise, the procedure was terminated by cardioversion.

\section{Surgical ablation procedure}

Ablation was carried out with bipolar Cardioablate (Medtronic, Minneapolis, MN) or an Atricure clamp (Atricure, West Chester, $\mathrm{OH}$ ) as we described previously [12]. After cross-clamping the aorta, the heart was arrested, and the LA was accessed through left atriotomy. The LA ablation line includes isolation of PVs and a connecting line was performed between both islands of PVs on the roof and a line from the left PVs to the posterior mitral annulus. The LAA was excised or excluded. Right atrial (RA) ablation line includes SVC to inferior vena cava; the lateral freewall lesion completes the anterior-medial tricuspid valve annulus; medial free-wall lesion completes the anteriormedial TV annulus and CTI ablation. The procedure was considered complete with the ablation of the right appendage.

\section{Tricuspid valve repair}

TV annuloplasty was subsequently performed with a Carpentier-Edwards MC3 ring (Edwards Lifesciences). The tricuspid ring was sized according to the combined surface area of the posterior and anterior tricuspid leaflets that are extended using a right-angle hook and implanted with simple, interrupted mattress sutures by sparing the septal annulus and conduction tissue in the region of the apex of the triangle of Koch.

\section{Post-procedure management}

Warfarin was given to maintain the international normalized ratio between 2.0 and 2.5 for the first 3 months to all patients. Patients who have no AF recurrence and a CHADS2 score $<2$ stopped taking warfarin 3 months following the procedure. Unless contraindicated, patients received amiodarone or sotalol within $24 \mathrm{~h}$ of the procedure, which were discontinued at 3 months (blanking period).

\section{Echocardiography}

Comprehensive 2-dimensional (2D) echocardiography was performed with a commercially available system (IE33; Philips Medical Systems, Andover, MA). Standard 2D and Doppler echocardiographic images were acquired in the left lateral decubitus position using a phased-array transducer in the parasternal and apical views by experienced cardiac sonographers. Three consecutive cardiac cycles were recorded and stored for subsequent analysis. Left ventricle end-diastolic (LVEDD) and end-systolic dimensions (LVESD) were measured from parasternal acquisitions. Left ventricle volumes and left ventricle ejection fraction (LVEF) were calculated using Simpson's biplane method according to the guidelines of the American Society of Echocardiography [13]. LA and RA areas were measured by planimetry at end-systole from the apical 4-chamber views. Left atrial volumes were measured by Simpson's biplane method. Color flow was applied in the apical 4chamber view to assess the severity of TR, which was then graded semiquantitatively based on a scale of 0 to 4 , where 0 , none or trace; $1+$, jet area/atrial area $<10 \%$ (mild TR); $2+$, jet area/atrial area 10 to $20 \%$ (moderate TR); $3+$, jet area/atrial area 20 to $33 \%$ (moderate-severe TR); and 4+, jet area/atrial area $>33 \%$ (severe TR) [14]. Tethering height was measured by tracing between the atrial surface of the leaflets and the tricuspid annular plane at the time of maximal systolic closure. From the apical 4-chamber view, the RV end-systolic and end-diastolic areas were measured by planimetry by positioning the transducer to maximize the $\mathrm{RV}$ area and to include the RV apex. RV fractional area change (RVFAC) was used to determine the RV systolic function and was calculated by the following formula: 
Table 1 Baseline characteristics before and after propensity-score matching

\begin{tabular}{|c|c|c|c|c|c|c|}
\hline & \multicolumn{3}{|l|}{ Pre-matching } & \multicolumn{3}{|l|}{ Post-matching } \\
\hline & Catheter $n=279$ & Surgical $n=114$ & SMD & Catheter $n=111$ & Surgical $n=111$ & SMD \\
\hline Age, yrs & $69.1 \pm 4.2$ & $68.3 \pm 4.8$ & 0.095 & $69.0 \pm 4.7$ & $68.8 \pm 4.5$ & 0.15 \\
\hline Male & $153(54.8)$ & $60(52.6)$ & 0.209 & $60(54.1)$ & $58(52.3)$ & -0.177 \\
\hline $\mathrm{BMI}, \mathrm{kg} / \mathrm{m}^{2}$ & $22.8 \pm 2.3$ & $22.7 \pm 2.7$ & 0.097 & $22.7 \pm 2.5$ & $22.7 \pm 2.5$ & 0.074 \\
\hline NYHA Class & $2.3 \pm 0.4$ & $2.5 \pm 0.6$ & 0.027 & $2.3 \pm 0.5$ & $2.3 \pm 0.5$ & 0.026 \\
\hline Smoking & $71(25.4)$ & $30(26.4)$ & -0.158 & $28(25.2)$ & $29(26.1)$ & -0.127 \\
\hline Diabetes & $29(10.4)$ & $13(11.4)$ & -0.202 & $12(10.8)$ & $12(10.8)$ & 0.049 \\
\hline Hypertension & $61(21.9)$ & $27(23.7)$ & -0.148 & $25(22.5)$ & $26(23.4)$ & -0.196 \\
\hline Stroke & $34(12.2)$ & $17(14.9)$ & 0.016 & $15(13.5)$ & $16(14.4)$ & 0 \\
\hline CAD & $31(11.1)$ & $15(13.2)$ & 0.003 & $14(12.6)$ & $14(12.6)$ & 0.081 \\
\hline COPD & $12(4.3)$ & $6(5.3)$ & 0.016 & $5(4.5)$ & $5(4.5)$ & -0.203 \\
\hline CHADS2 Score & $0.68 \pm 1.32$ & $0.70 \pm 1.82$ & 0.058 & $0.69 \pm 1.19$ & $0.70 \pm 1.23$ & 0.076 \\
\hline Euro SCORE & $0.75 \pm 1.02$ & $0.79 \pm 1.10$ & -0.175 & $0.76 \pm 1.09$ & $0.78 \pm 1.12$ & -0.274 \\
\hline AF duration, years & $2.5 \pm 1.1$ & $3.3 \pm 1.6$ & 0.169 & $2.5 \pm 1.2$ & $2.6 \pm 1.3$ & 0 \\
\hline \multicolumn{7}{|l|}{ Medication } \\
\hline ACE I & $51(18.2)$ & 22 (19.3) & 0.009 & 21 (18.9) & 21 (18.9) & 0.155 \\
\hline ARB & $27(9.7)$ & $13(11.4)$ & -0.011 & $11(10.0)$ & $12(10.8)$ & 0.176 \\
\hline$C C B$ & $20(7.1)$ & $11(9.6)$ & -0.007 & $9(8.1)$ & $10(9.0)$ & -0.137 \\
\hline Beta-blocker & 52 (18.6) & $14(12.3)$ & 0.136 & 15 (13.5) & 14 (12.6) & -0.127 \\
\hline Digitalis & $78(28.0)$ & $22(19.3)$ & 0.144 & 24 (21.6) & $22(19.8)$ & -0.156 \\
\hline Diuretics & $83(29.7)$ & $24(21.1)$ & 0.113 & $26(23.4)$ & 24 (21.6) & -0.121 \\
\hline Amiodarone & $100(35.8)$ & $30(26.3)$ & -0.421 & $32(28.8)$ & $30(27.0)$ & -0.17 \\
\hline LVESD, mm & $36.1 \pm 10.1$ & $37.5 \pm 11.6$ & 0.013 & $36.9 \pm 11.5$ & $37.1 \pm 10.8$ & 0.105 \\
\hline LVEDD, mm & $52.1 \pm 5.8$ & $53.7 \pm 6.8$ & -0.163 & $52.8 \pm 6.1$ & $53.0 \pm 6.3$ & 0.161 \\
\hline sPAP, mmHg & $39.1 \pm 9.2$ & $40.7 \pm 6.2$ & -0.184 & $39.8 \pm 8.9$ & $40.0 \pm 7.1$ & 0.125 \\
\hline $\mathrm{LAD}, \mathrm{mm}$ & $52.1 \pm 11.7$ & $53.6 \pm 10.8$ & -0.011 & $52.6 \pm 10.3$ & $52.9 \pm 10.9$ & -0.055 \\
\hline LVEF, \% & $60.1 \pm 8.2$ & $58.6 \pm 9.2$ & -0.127 & $59.6 \pm 8.9$ & $59.1 \pm 9.0$ & 0.071 \\
\hline RAA, $\mathrm{mm}^{2}$ & $19.7 \pm 5.1$ & $20.9 \pm 3.5$ & -0.164 & $20.0 \pm 4.9$ & $20.1 \pm 4.9$ & 0.219 \\
\hline RVSI & $2.0 \pm 0.2$ & $2.0 \pm 0.5$ & -0.162 & $2.0 \pm 0.3$ & $2.0 \pm 0.3$ & 0.165 \\
\hline RVFAC, \% & $43.1 \pm 4.8$ & $42.2 \pm 6.7$ & -0.243 & $42.7 \pm 4.3$ & $42.4 \pm 5.1$ & -0.042 \\
\hline Tethering height, mm & $7.1 \pm 3.6$ & $8.9 \pm 3.9$ & -0.294 & $7.9 \pm 3.4$ & $8.2 \pm 3.7$ & 0.017 \\
\hline Tethering area, $\mathrm{cm}^{2}$ & $2.8 \pm 0.8$ & $3.7 \pm 0.7$ & 0.217 & $2.9 \pm 0.9$ & $3.2 \pm 0.5$ & 0 \\
\hline TR EROA, $\mathrm{cm}^{2}$ & $0.8 \pm 0.4$ & $0.9 \pm 0.8$ & -0.127 & $0.8 \pm 0.5$ & $0.8 \pm 0.7$ & -0.157 \\
\hline $\mathrm{TR} V \mathrm{C}, \mathrm{cm}$ & $1.0 \pm 0.4$ & $1.0 \pm 0.7$ & -0.133 & $1.0 \pm 0.2$ & $1.0 \pm 0.3$ & 0.075 \\
\hline TR grade & $3.4 \pm 0.7$ & $3.7 \pm 0.5$ & 0.022 & $3.5 \pm 0.5$ & $3.6 \pm 0.2$ & -0.095 \\
\hline $\mathrm{TAD}, \mathrm{cm}$ & $36.5 \pm 4.1$ & $39.1 \pm 5.2$ & 0.003 & $37.9 \pm 4.9$ & $38.8 \pm 5.0$ & 0.081 \\
\hline
\end{tabular}

ACEl Angiotensin-converting enzyme inhibitor, $A F$ Atrial fibrillation, $A R B$ Angiotensin receptor blocker; $B M I$ Body mass index, $C A D$ Coronary artery disease, $C C B$ Calcium channel blocker, COPD Chronic obstructive pulmonary disease, EROA Effective regurgitant orifice area, $L A D$ Left atrial diameter, $L V E F$ Left ventricle ejection fraction, LVEDD Left ventricle end-diastolic dimension, LVESD Left ventricle end-systolic dimension, NYHA New York Heart Association, RAA Right atrial area, RVFAC Right ventricle fractional area change, RVSI Right ventricle sphericity index, SMD Standard mean diference, sPAP Pulmonary artery systolic pressure, TAD Tricuspid annulus diameter, TR Tricuspid regurgitation, VC Vena contracta

$\mathrm{FAC}=([$ diastolic area systolic area $] /$ diastolic area $) \times 100 \%$ [15]. RV long-axis length and RV short-axis width at the midventricular level were measured as described by Matsunaga and Duran and used to calculate the end-diastolic RV sphericity index (RVSI) as previously described (RVSI $=\mathrm{RV}$ long-axis length/RV short-axis width) [16]. Systolic pulmonary artery pressure (sPAP) was measured by echocardiography using the modified Bernoulli equation on the transtricuspid continuous-wave Doppler signal, while adding pressure on RA. The TV tethering height and TV 
tethering area were measured by tracing between the atrial surface of the leaflets and the tricuspid annular plane at the end-systole.

\section{Patient follow-up}

All patients had visits scheduled at 1, 3, and 12 months postoperatively and every 1 year thereafter. At each visit, the patient's history, physical examination, chest X-ray, a 24-h Holter, and echocardiogram were performed and obtained. The earliest echocardiogram on which moderate or greater TR was indicated for each patient was used to designate recurrent TR.

\section{Statistics}

All continuous variables are presented as mean standard deviation (SD), categorical values are presented as percentages, and odds ratios are presented with $95 \%$ confidence intervals (CIs). Comparisons between surgical and catheter cohorts were done by $t$ test for continuous variables, chi-square test for categorical variables. A univariate and multivariate Cox hazard regression analysis was used to identify predictors of survival and freedom from events. The optimal cut-off value of the parameters in predicting the recurrence was identified using receiver operating characteristic (ROC) curve analysis. The area under the ROC curve (AUC) was calculated and compared by the DeLong method. Variables selected to be tested on multivariate analysis included those with $P<$ 0.1 on univariate analysis. To reduce the effect of potential confounding factors in subgroup analysis, propensity-score match (PSM) analysis was used. The analysis was performed by matching patients in the 2 groups at a 1:1 ratio, without replacement, by the nearest neighbor technique, using a caliper width equal to 0.2 of the SD of the logit of the propensity score. A competing risk regression model was used to assess AF recurrence and stroke with death as a competing event. All significance tests were 2-tailed, and $p$ value of $<0.05$ was considered to be statistically significant. All analyses were performed with Stata/SE version 15 (Stata Corporation, Lakeway Drive College Station, TX, USA).

\section{Results}

\section{Patients}

There was a total of 1916 patients who underwent first catheter or surgical ablation between January 2008 and December 2013. There were only 411 patients that met the study criteria after review. Of these, 132 were treated by surgical ablation and TV repair, and 114 of whom had no history of left-sided valve surgery were included as the surgical cohort. Of the remaining 279 patients who were treated with '2C3L' catheter ablation were selected as the catheter cohort. Baseline characteristics were shown in Table 1.

\section{Procedural outcomes}

All patients successfully underwent the procedure, and there was no in-hospital mortality. In both cohorts, $100 \%$ PVs that are targeted for ablation demonstrated entrance and exit block at the conclusion of the procedure. Intraprocedural AF termination was more (Table 2). In catheter cohort, the mean procedural time, fluoroscopy time and radiofrequency time were $215 \pm 38 \mathrm{~min}, 48 \pm 11 \mathrm{~min}$ and $116 \pm 24 \mathrm{~min}$, respectively. In surgical cohort, the mean duration of cardiopulmonary bypass and aortic crossclamping was $97 \pm 24 \mathrm{~min}$ and $46 \pm 20 \mathrm{~min}$, respectively.

\section{Outcomes during follow-up}

All included patients have completed a 5-year clinical follow-up. After a mean follow-up period of 77 months, there were 22 and 12 deaths in the catheter and surgical cohort (Table 3). During the follow-up, only 1 patient required pacemaker implantation for sinus node dysfunction in surgical cohort. On Kaplan-Meier analysis, the estimated actuarial 5-year survival rates were 96.8\% (CI: 92.95-97.78) and 92.0\% (CI: 85.26-95.78) in the catheter and surgical cohort, respectively (Fig. 2a). In contrast to the surgical cohort, the catheter cohort had a higher risk of stroke (SHR: 0.3790; CI: 0.180-0.797; $P=0.011$, Fig. 2c), but had a similar risk of recurrent AF (SHR: 0.8458; CI: $0.637-1.124 ; P=0.248$, Fig. $2 \mathrm{~b}$ ) and moderate-severe TR (SHR: 1.2950; CI: 0.924-1.814; $P=0.133$, Fig. $2 \mathrm{~d}$ ) after considering death as a competing risk.

\section{Heart rhythm and echocardiography during follow-up}

All patients in both cohorts had their complete Holter, and echocardiogram follow-up results. According to the rhythm status at follow-up, study population were divided into the patients with sinus rhythm (SR) and with recurrent $\mathrm{AF}$ in each cohort.

In catheter cohort, at baseline, both groups had similar percentages of patients with moderate-severe TR. At follow-up, $13 \%$ of patients with SR had trace or no TR,

Table 2 Details of the procedures

\begin{tabular}{|c|c|c|c|}
\hline Procedure parameters & $\begin{array}{l}\text { Catheter cohort } \\
n=279\end{array}$ & $\begin{array}{l}\text { Surgical cohort } \\
n=114\end{array}$ & $P$ value \\
\hline $\begin{array}{l}\text { AF termination rate, } \\
\mathrm{n}(\%)\end{array}$ & $145(51.9)$ & $90(78.9)$ & $<0.0001$ \\
\hline $\begin{array}{l}\text { PVs entrance and } \\
\text { exit block, } n(\%)\end{array}$ & $279(100)$ & $114(100)$ & NS \\
\hline $\begin{array}{l}\text { Cardiac tamponade, } \\
\mathrm{n}(\%)\end{array}$ & $2(0.7)$ & $0(0)$ & NS \\
\hline $\begin{array}{l}\text { Reexploration for } \\
\text { bleeding, n (\%) }\end{array}$ & $0(0)$ & $1(0.9)$ & NS \\
\hline $\begin{array}{l}\text { AKI requiring dialysis, } \\
\mathrm{n}(\%)\end{array}$ & $0(0)$ & $1(0.9)$ & NS \\
\hline CABG & 0 & $5(4.4)$ & 0.060 \\
\hline
\end{tabular}

$A F$ Atrial fibrillation, $A K I$ Acute kidney injury, CABG Coronary artery bypass graft, PVs Pulmonary veins 
Table 3 Causes of Death during Follow-up

\begin{tabular}{llll}
\hline & Catheter cohort $\boldsymbol{n = 2 7 9}$ & Surgical cohort $\boldsymbol{n = 1 1 4}$ & $\boldsymbol{P}$ value \\
\hline Congestive heart failure, $\mathrm{n}(\%)$ & $10(3.6)$ & $9(7.9)$ & 0.373 \\
Acute myocardial infarction, $\mathrm{n}(\%)$ & $1(0.4)$ & $0(0)$ & NS \\
Stroke, $\mathrm{n}(\%)$ & $5(1.8)$ & $1(0.9)$ & NS \\
Malignancy, $\mathrm{n}(\%)$ & $2(0.7)$ & $0(00)$ & NS \\
Sudden cardiac death, $\mathrm{n}(\%)$ & $2(0.7)$ & $1(0.9)$ & NS \\
Unknown cause, $\mathrm{n}(\%)$ & $2(0.7)$ & $1(0.9)$ & NS \\
\hline
\end{tabular}

compared with $0 \%$ in the recurrent group, and $69 \%$ with SR had mild TR compared with $32 \%$ in the recurrent group. Only $18 \%$ of patients with SR still had moderatesevere TR at follow-up, compared with $68 \%$ in the recurrent group ( $p<0.0001$ for entire trend) (Fig. 3a). Moreover, SR group had a significant decrease in tethering height, tethering area, TR effective regurgitant orifice area (EROA), TR vena contracta (VC), TR grade and tricuspid annular dimension (TAD), with RV sphericity index (RVSI) and RV fractional area change (RVFAC) significantly increased at follow-up. And the recurrent group had a significant increase in right atrial (RA) area, TR EROA, TR grade and TAD, with RVSI and RVFAC significantly decreased at follow-up (Supplementary Table 1).

In surgical cohort, at baseline, both groups had similar percentages of patients with moderate-severe TR. At follow-up, all patients with recurrent AF had moderatesevere TR at follow-up when compared with $33 \%$ in the SR group ( $p<0.0001$ for entire trend) (Fig. 3b). Moreover,

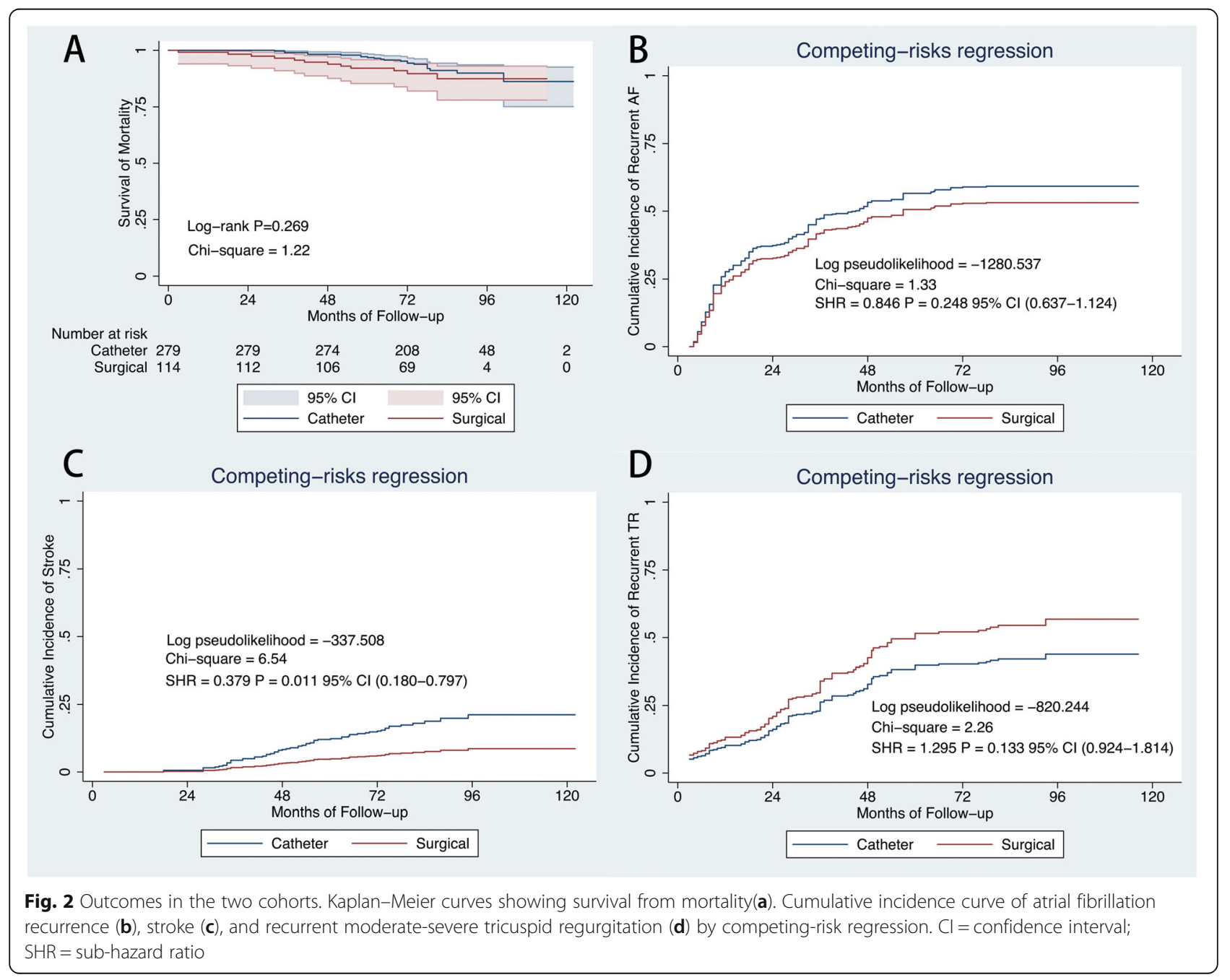




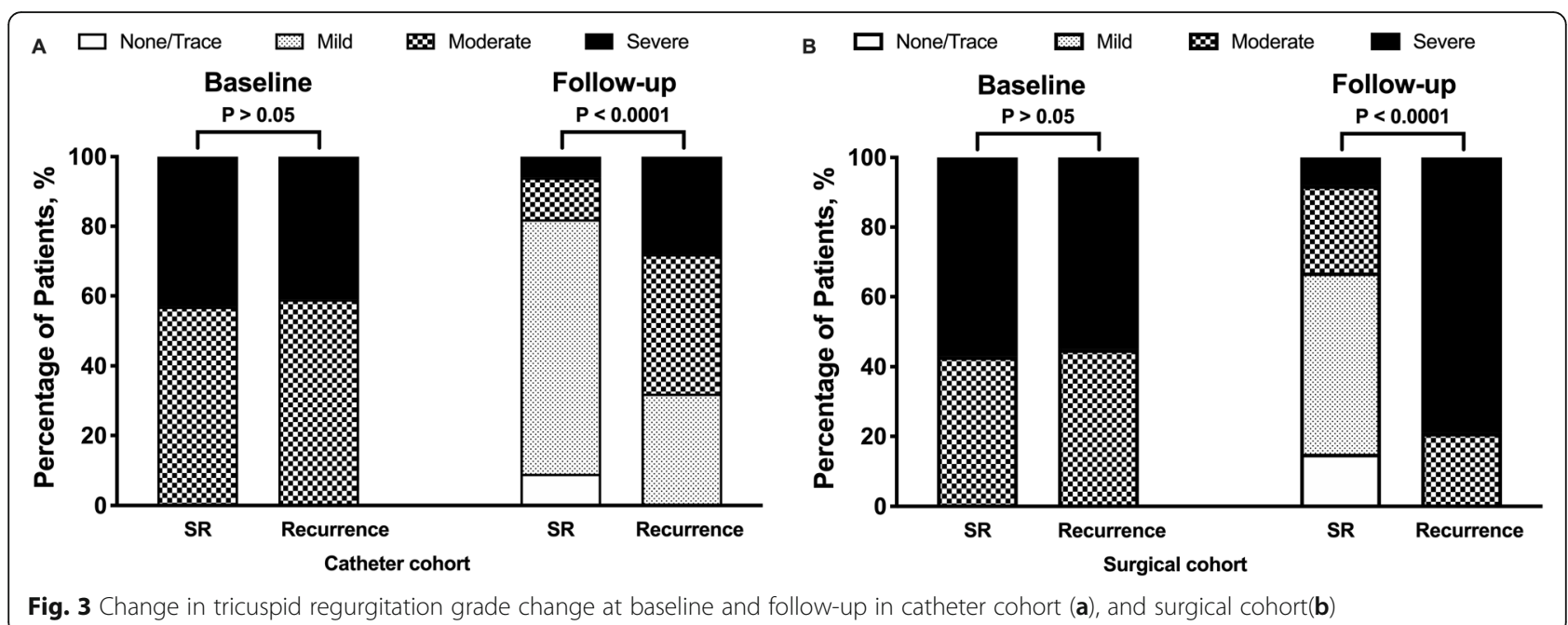

Table 4 Predictors of recurrence of AF in catheter and surgical cohorts: univariate and multivariate analyses

\begin{tabular}{|c|c|c|c|c|c|}
\hline & & \multicolumn{2}{|l|}{ Univariate } & \multicolumn{2}{|l|}{ Multivariate } \\
\hline & & $\mathrm{HR}(95 \% \mathrm{Cl})$ & $P$ value & $\mathrm{HR}(95 \% \mathrm{Cl})$ & $P$ value \\
\hline \multirow[t]{2}{*}{ Age (per 10 years) } & Catheter & $3.12(1.32-6.72)$ & 0.048 & $2.90(0.75-5.65)$ & 0.184 \\
\hline & Surgical & $3.34(1.49-6.49)$ & 0.020 & $3.01(0.86-5.76)$ & 0.152 \\
\hline \multirow[t]{2}{*}{ CHADS2 Score } & Catheter & $4.12(0.76-9.06)$ & 0.079 & $3.17(0.98-8.42)$ & 0.801 \\
\hline & Surgical & $3.39(0.59-6.49)$ & 0.077 & $2.50(0.73-4.21)$ & 0.199 \\
\hline \multirow[t]{2}{*}{ AF duration, months } & Catheter & $4.02(1.16-8.89)$ & 0.014 & $3.41(0.94-6.19)$ & 0.461 \\
\hline & Surgical & $4.86(1.09-8.48)$ & 0.037 & $3.71(0.29-6.48)$ & 0.303 \\
\hline \multirow[t]{2}{*}{ LAD, mm } & Catheter & $1.05(0.18-2.59)$ & 0.097 & $0.97(0.25-1.62)$ & 0.344 \\
\hline & Surgical & $1.46(0.59-2.51)$ & 0.065 & $1.10(0.37-1.75)$ & 0.290 \\
\hline \multirow[t]{2}{*}{ RAA, $\mathrm{mm}^{2}$} & Catheter & $2.40(1.50-5.26)$ & 0.021 & $1.01(0.32-3.39)$ & 0.053 \\
\hline & Surgical & $3.50(1.69-8.03)$ & 0.015 & $2.98(0.79-8.23)$ & 0.815 \\
\hline \multirow[t]{2}{*}{ RVSI } & Catheter & $3.20(1.20-7.13)$ & 0.007 & $3.11(1.69-6.76)$ & 0.023 \\
\hline & Surgical & $2.94(1.63-6.60)$ & 0.002 & $3.01(1.27-5.23)$ & 0.004 \\
\hline \multirow[t]{2}{*}{ RVFAC, \% } & Catheter & $3.01(1.40-5.38)$ & 0.021 & $1.31(0.35-2.81)$ & 0.076 \\
\hline & Surgical & $1.47(1.04-3.19)$ & 0.016 & $1.19(0.23-2.69)$ & 0.110 \\
\hline \multirow[t]{2}{*}{ Tethering height, mm } & Catheter & $5.32(1.79-10.04)$ & 0.017 & $4.06(2.15-5.69)$ & 0.011 \\
\hline & Surgical & $4.62(1.58-9.83)$ & 0.011 & $3.82(1.92-5.42)$ & 0.035 \\
\hline \multirow[t]{2}{*}{ Tethering area, $\mathrm{cm}^{2}$} & Catheter & $1.52(1.06-4.25)$ & 0.008 & $0.97(0.30-1.46)$ & 0.144 \\
\hline & Surgical & $2.50(1.66-6.39)$ & 0.033 & $1.53(0.73-2.21)$ & 0.178 \\
\hline \multirow[t]{2}{*}{ TR EROA, $\mathrm{cm}^{2}$} & Catheter & $3.53(1.39-7.30)$ & 0.028 & $1.95(0.78-5.88)$ & 0.082 \\
\hline & Surgical & $2.49(1.37-6.38)$ & 0.025 & $1.85(0.05-3.23)$ & 0.121 \\
\hline \multirow[t]{2}{*}{ TR VC, cm } & Catheter & $2.39(0.35-6.59)$ & 0.078 & $2.52(0.50-5.04)$ & 0.127 \\
\hline & Surgical & $2.28(0.18-6.18)$ & 0.082 & $1.43(0.26-5.37)$ & 0.262 \\
\hline \multirow[t]{2}{*}{ TR grade } & Catheter & $2.05(1.58-5.33)$ & 0.019 & $1.97(0.69-3.26)$ & 0.099 \\
\hline & Surgical & $1.95(1.01-5.21)$ & 0.037 & $2.29(0.98-3.58)$ & 0.194 \\
\hline \multirow[t]{2}{*}{ TAD, cm } & Catheter & $2.54(1.19-5.95)$ & 0.016 & $1.90(0.76-4.58)$ & 0.415 \\
\hline & Surgical & $2.11(1.57-5.52)$ & 0.039 & $1.66(0.52-4.34)$ & 0.555 \\
\hline
\end{tabular}

AF Atrial fibrillation, $\mathrm{Cl}=\mathrm{Cl}$ Confidence interval, EROA Effective regurgitant orifice area, $L A D$ Left atrial diameter, RAA Right atrial area, RVFAC Right ventricle fractional area change, RVSI Right ventricle sphericity index, TR Tricuspid regurgitation, VC Vena contracta 
SR group had a significant decrease in tethering height, tethering area, TR EROA, TR VC, TR grade and TAD, with RVSI and RVFAC significantly increased at followup. And the recurrent group had a significant increase in RA area and TAD, with RVSI and RVFAC significantly decreased at follow-up. (Supplementary Table 2).

\section{Predictor for the recurrence of either AF or moderate-severe $T R$}

Multivariate Cox hazard regression analysis revealed that RVSI and tethering height as independent risk factors for the recurrence of either AF or moderate-severe TR in both cohorts (Tables 4 and 5). Next, the optimal cut-off value of the RVSI and tethering height in predicting the recurrence was identified using ROC curve analysis (Table 6). Interestingly, the tethering height showed a significantly better predictive performance than RVSI in the recurrence of either AF or moderate-severe TR in both cohorts (Fig. 4).
According to the merits of tethering height mentioned above, we made a 3-D plot graph to demonstrate the relation between basal tethering height, change in TR severity at follow-up and SR restored duration after the procedure in total cohort (Fig. 5). It showed the higher the basal tethering height was, the more changes in TR severity at follow-up were, and the less SR restored duration after the procedure was.

\section{Propensity-score match analysis between two cohorts}

A propensity-score matching was performed to adjust for differences in measured baseline characteristics between 2 cohorts (Table 1, Supplementary Figure 1). Among the 111 PSM pairs, the catheter group showed significantly higher rates of stroke $(\mathrm{SHR}=0.286 ; 95 \% \mathrm{CI}$, $0.106-0.768 ; P=0.013)$, and AF recurrence $(\mathrm{SHR}=$ 0.669; 95\% CI, 0.486-0.976; $P=0.036$ ) after considering death as a competing risk (Fig. 6). We categorized matched patients into subgroups by tethering height $<6$

Table 5 Predictors of recurrence of TR in catheter and surgical cohort: univariate and multivariate analyses

\begin{tabular}{|c|c|c|c|c|c|}
\hline & & \multicolumn{2}{|l|}{ Univariate } & \multicolumn{2}{|l|}{ Multivariate } \\
\hline & & HR (95\% Cl) & $P$ value & HR (95\% Cl) & $P$ value \\
\hline \multirow[t]{2}{*}{ Age (per 10 years) } & Catheter & $2.98(1.44-5.82)$ & 0.051 & $4.30(0.50-8.30)$ & 0.092 \\
\hline & Surgical & $2.11(1.69-6.36)$ & 0.065 & $3.83(0.94-7.69)$ & 0.210 \\
\hline \multirow[t]{2}{*}{ AF duration, months } & Catheter & $2.90(1.04-4.86)$ & 0.016 & $2.01(0.73-4.29)$ & 0.450 \\
\hline & Surgical & $2.19(1.71-6.94)$ & 0.044 & $2.38(0.83-5.12)$ & 0.167 \\
\hline \multirow[t]{2}{*}{$L A D, m m$} & Catheter & $3.72(0.50-8.61)$ & 0.085 & $3.51(0.94-7.23)$ & 0.369 \\
\hline & Surgical & $2.57(0.98-5.18)$ & 0.078 & $4.27(0.63-6.17)$ & 0.137 \\
\hline \multirow[t]{2}{*}{ RAA, $\mathrm{mm}^{2}$} & Catheter & $4.63(1.74-9.56)$ & 0.042 & $4.36(0.52-8.05)$ & 0.254 \\
\hline & Surgical & $4.22(0.91-8.52)$ & 0.051 & $5.54(0.10-7.24)$ & 0.080 \\
\hline \multirow[t]{2}{*}{ RVSI } & Catheter & $3.63(1.62-8.30)$ & 0.004 & $3.13(1.91-6.41)$ & 0.009 \\
\hline & Surgical & $3.18(1.50-7.88)$ & 0.005 & $3.21(1.99-6.49)$ & 0.015 \\
\hline \multirow[t]{2}{*}{ RVFAC, \% } & Catheter & $3.16(1.43-7.76)$ & 0.039 & $3.03(0.84-4.47)$ & 0.065 \\
\hline & Surgical & $3.05(0.93-6.36)$ & 0.060 & $2.31(0.12-3.75)$ & 0.063 \\
\hline \multirow[t]{2}{*}{ Tethering height, mm } & Catheter & $3.16(1.17-7.30)$ & 0.008 & $3.00(1.69-5.56)$ & 0.004 \\
\hline & Surgical & $3.14(1.35-8.38)$ & 0.001 & $4.01(2.71-6.58)$ & 0.009 \\
\hline \multirow[t]{2}{*}{ Tethering area, $\mathrm{cm}^{2}$} & Catheter & $2.60(0.92-4.68)$ & 0.079 & $1.38(0.52-2.93)$ & 0.063 \\
\hline & Surgical & $1.49(1.74-3.65)$ & 0.028 & $1.46(0.89-3.30)$ & 0.084 \\
\hline \multirow[t]{2}{*}{ TR EROA, $\mathrm{cm}^{2}$} & Catheter & $2.96(0.61-6.56)$ & 0.058 & $1.33(0.84-4.62)$ & 0.082 \\
\hline & Surgical & $1.56(0.92-5.53)$ & 0.059 & $1.11(0.61-4.39)$ & 0.065 \\
\hline \multirow[t]{2}{*}{ TR VC, cm } & Catheter & $2.55(0.86-5.60)$ & 0.073 & $3.53(0.84-7.55)$ & 0.096 \\
\hline & Surgical & $3.32(0.72-7.96)$ & 0.092 & $3.21(0.50-7.19)$ & 0.365 \\
\hline \multirow[t]{2}{*}{ TR grade } & Catheter & $3.08(1.47-7.80)$ & 0.032 & $3.17(1.17-7.37)$ & 0.102 \\
\hline & Surgical & $3.1(0.92-6.52)$ & 0.072 & $2.68(1.48-6.04)$ & 0.068 \\
\hline \multirow[t]{2}{*}{ TAD, cm } & Catheter & $3.61(0.74-7.17)$ & 0.098 & $2.79(0.50-6.88)$ & 0.208 \\
\hline & Surgical & $3.96(0.68-9.47)$ & 0.071 & $3.01(0.32-8.01)$ & 0.628 \\
\hline
\end{tabular}

AF Atrial fibrillation, $\mathrm{Cl}=\mathrm{Cl}$ Confidence interval, EROA Effective regurgitant orifice area, $L A D$ Left atrial diameter, $R A A$ Right atrial area, $R V F A C$ Right ventricle fractional area change, RVSI Right ventricle sphericity index, TR Tricuspid regurgitation, VC Vena contracta 


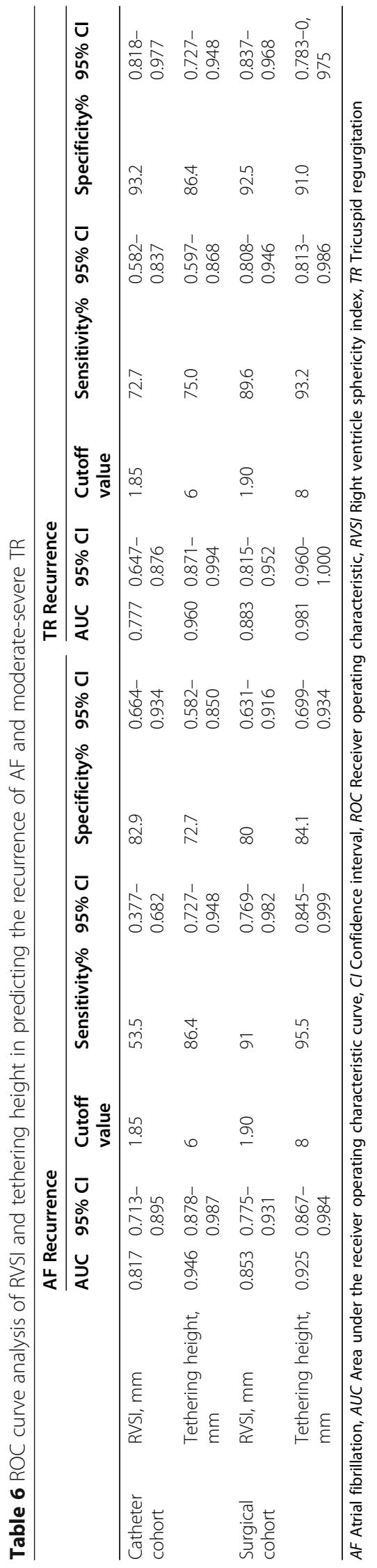



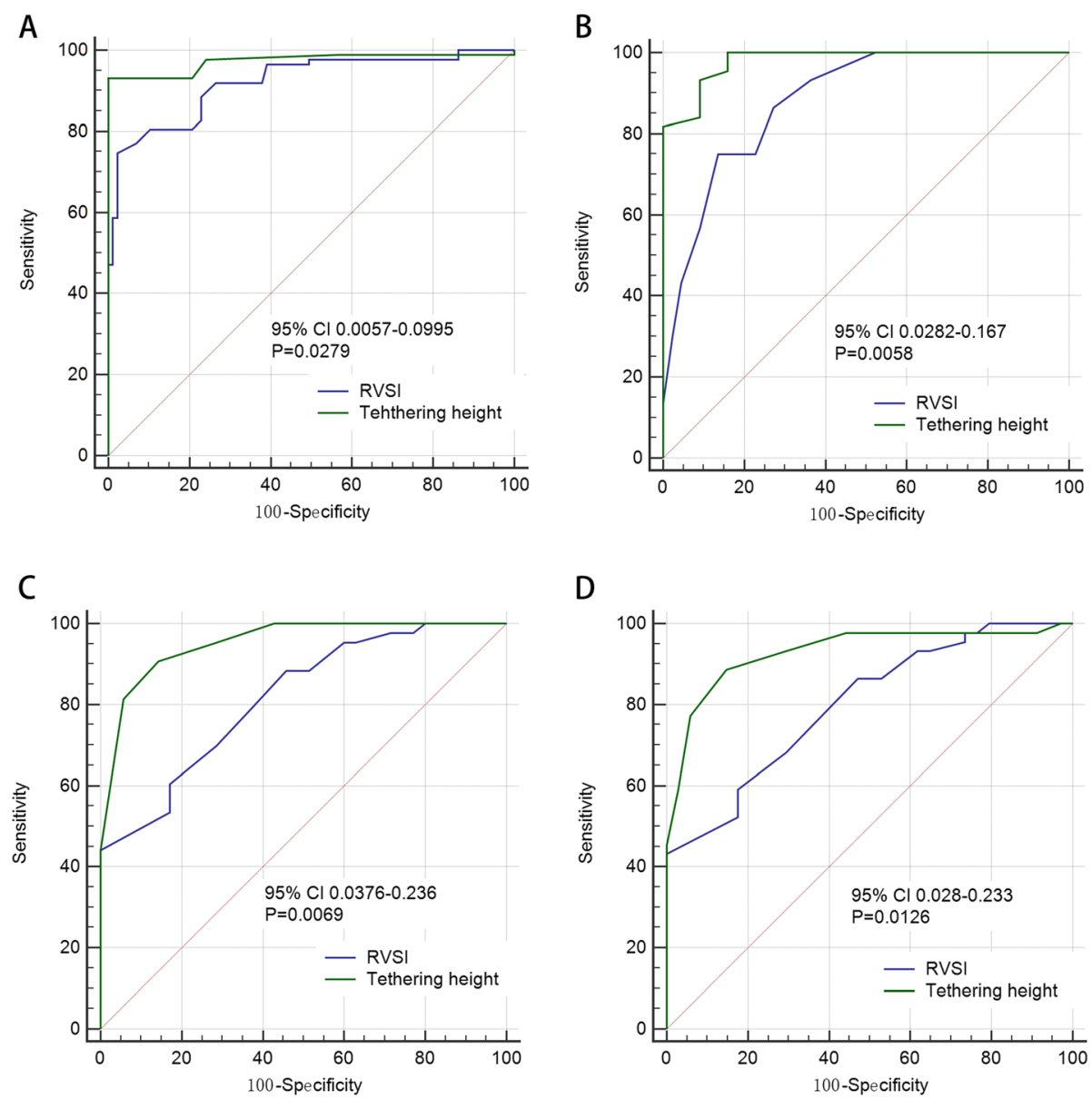

Fig. 4 Comparing the areas under ROC curve of tethering height, and RVSI for the prediction of recurrent atrial fibrillation in catheter cohort (a) and surgical cohort (c); and for the prediction of recurrent moderate-severe tricuspid regurgitation in catheter cohort (b) and surgical cohort (d). $\mathrm{Cl}=$ confidence interval

$\mathrm{mm}$ and $\geq 6 \mathrm{~mm}$ to determine whether a graded relation exists with tethering height and our further customizing and selecting the best treatment options for the patients with AF-caused moderate-severe TR. On Kaplan-Meier analysis, there were no difference in survival from mortality in patients with tethering height $<6 \mathrm{~mm}$ and $\geq 6 \mathrm{~mm}$ (Figs. 7a, 8a). On competing risk analysis, the cumulative incidence of stroke, recurrent $\mathrm{AF}$ and moderate-severe TR showed no difference between two groups (Fig. 7 b-d). However, the cumulative incidence of stroke (SHR $=0.199 ; 95 \%$ CI, 0.047-0.849; $P=0.029)$, recurrent AF $(\mathrm{SHR}=$ 0.589 ; 95\% CI, $0.357-09738 ; P=0.039)$ and moderatesevere TR $(\mathrm{SHR}=0.593 ; 95 \% \mathrm{CI}, 0.359-0.981 ; P=$ 0.042) were higher for patients with tethering height $\geq 6 \mathrm{~mm}$ in catheter group (Fig. 8 b-d). Examples of two patients in each cohort with different TR severity at follow-up are shown in Supplementary Figure 2 and 3.

\section{Discussion}

According to our knowledge, this is the first study to compare the mid-term follow-up outcomes of catheter and surgical treatments in patients with LSPAF and moderate-severe functional TR. The main findings showed that (i) catheter ablation without intervention for TV seemed to be an optional treatment in patients with less TV tethering, achieving satisfied SR restored rate and TR improvement; (ii) whereas patients with less leaflets coaptation might benefit more from surgical ablation and TV repair to achieve better SR restored rate and less TR deterioration than catheter ablation.

Gould et al. first suggested that if AF increased the end-diastolic volume above a critical level, then it resulted in TR [17]. Isolated tricuspid annular dilatation that is related to RA enlargement might not be enough to cause severe functional TR in patients with AF, although most of the studies reported that tricuspid 


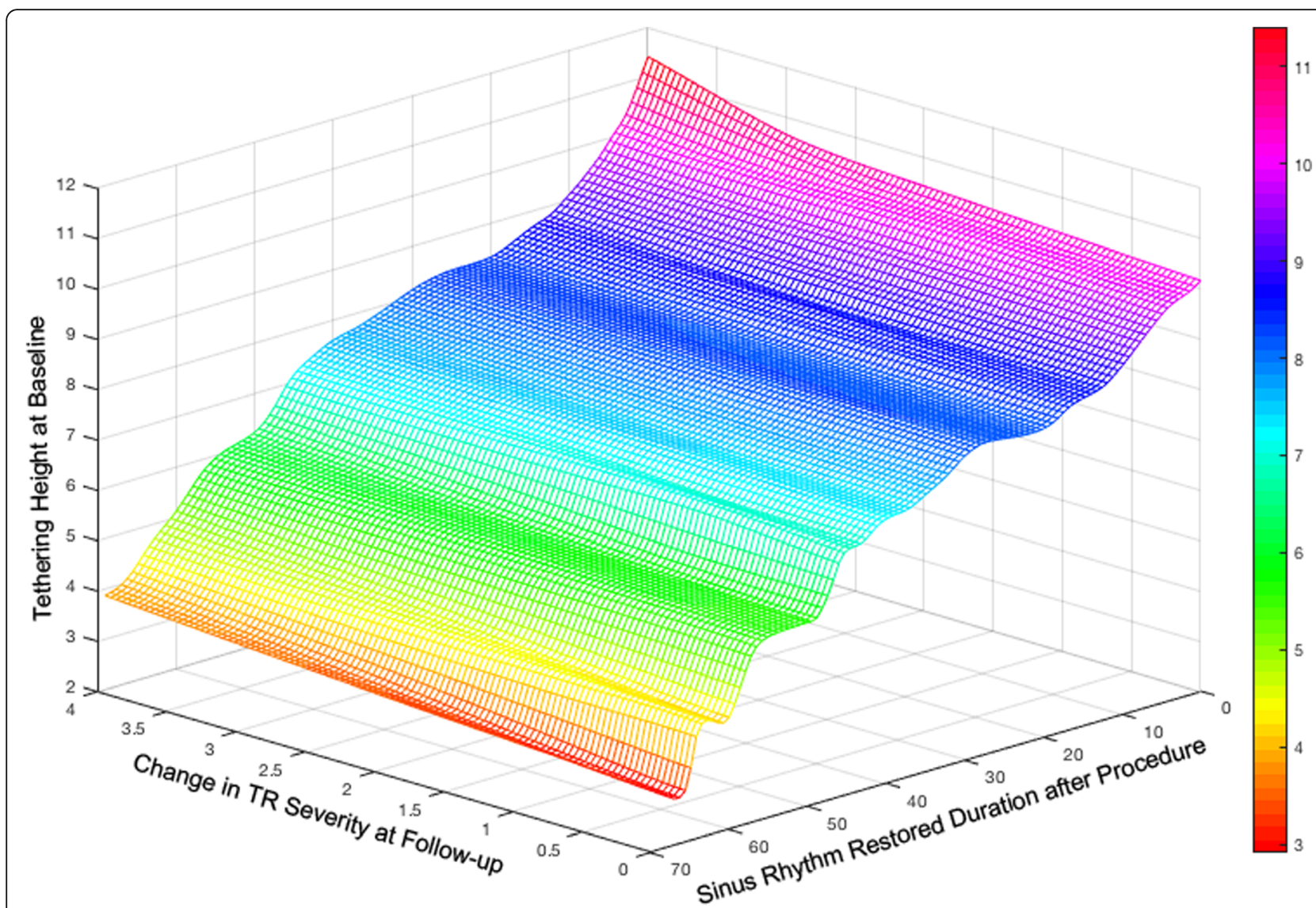

Fig. 5 Relation between basal tethering height, change in tricuspid regurgitation severity at follow-up and sinus rhythm restored duration after the procedure

annular dilatation is related to RA volume. Further tricuspid annular dilatation with RV enlargement and remodeling might develop into severe TR, leading to an altered ventricular force balance to close the leaflets and incomplete closure of the TVs. It has been reported that RV eccentric dilatation and tethering of leaflets might be more important in patients with severe TR than dilatation of tricuspid annular $[18,19]$. These results revealed that TRAF occurred secondary to right-sided heart remodeling, TV deformations and annular dilatation, which was consistent with the present study results. Existing dysfunction or remodeling of RV can act as a substrate for AF occurrence as well. So, AF caused RV remodeling can beget $\mathrm{TR}$, which in turn causes $\mathrm{AF}$ further.

The importance of heart rhythm in RV functioning is well known $[20,21]$. The RV ejection fraction in patients with AF was deteriorated compared to healthy individuals. AF may lead to RV dysfunction and remodeling, which subsequently caused papillary muscle displacement, tethering of TV leaflet, tricuspid annular dilation, and TR. In our study, we assessed the clinical and echocardiographic parameters that contributed to AF and TR progression after ablation, and the results revealed that RVSI and tethering height were both independent risk factors for the recurrence of either AF or moderate-severe TR. Moreover, ROC analysis suggested that tethering height was a stronger predictor than RVSI in the recurrence of either AF or moderate-severe TR. The higher the basal tethering height was, the more changes in the TR severity at follow-up were, and the less SR restored duration after the procedure was. The results indirectly indicated that right-sided heart remodeling was strongly associated with rhythm status as well as a vicious cycle, thereby leading to worsened TR that progress over time.

Our PSM analysis demonstrates that the cumulative incidence of recurrent $\mathrm{AF}$ and stroke in surgical group were lower than those in catheter group. It demonstrated that besides improving efficacy, thromboembolic risk can be further decreased by 


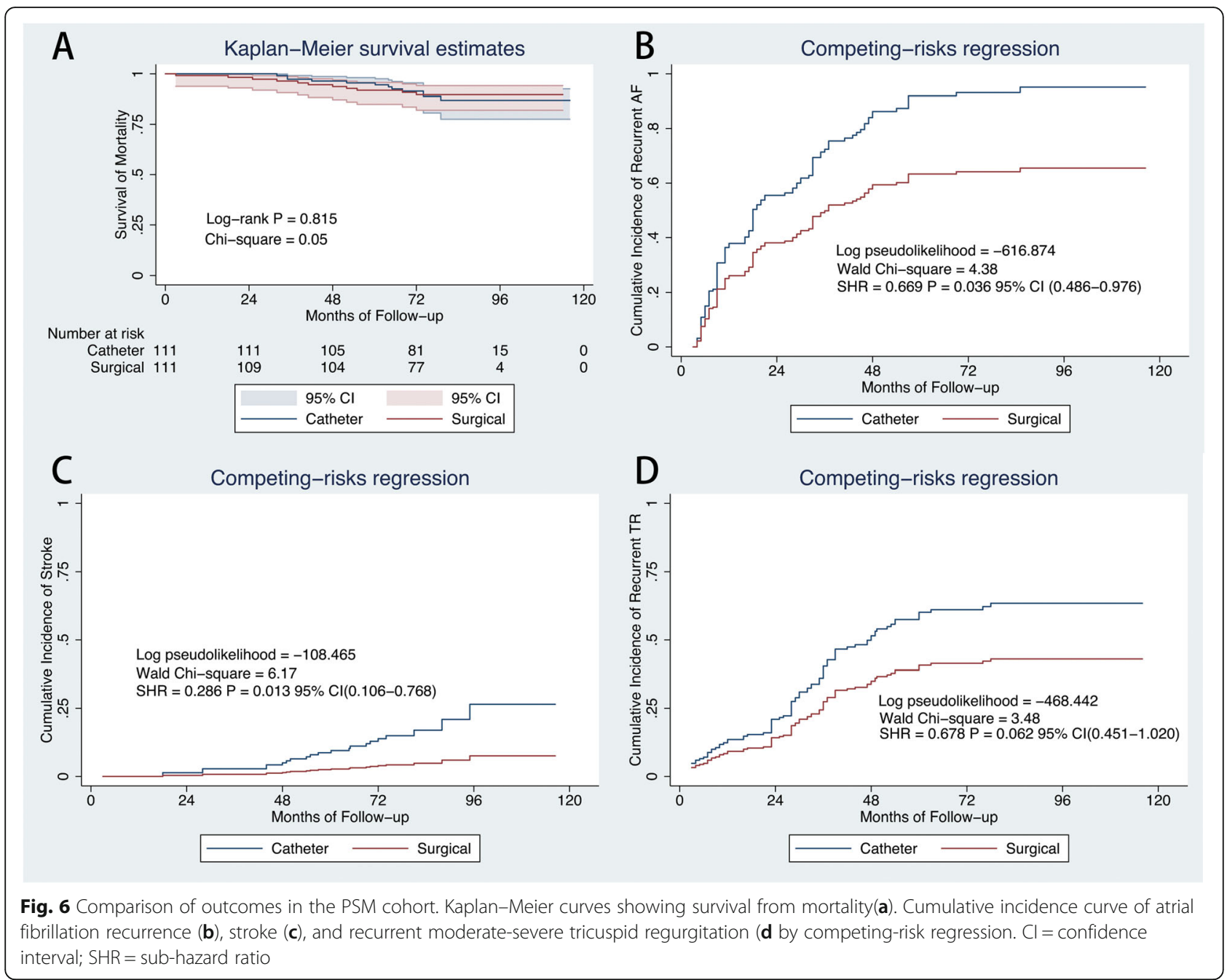

exclusion of the left atrial appendage. But after categorized matched patients into subgroups by tethering height $<6 \mathrm{~mm}$ and $\geq 6 \mathrm{~mm}$, we found that in patients with tethering height $<6 \mathrm{~mm}$, there was no difference in the cumulative incidence of recurrent $\mathrm{AF}$ and moderate-severe TR between catheter ablation and surgical treatment. It suggested that that the AFcaused moderate-severe TR could be potentially treated with ' $2 \mathrm{C} 3 \mathrm{~L}$ ' catheter ablation in patients with tethering height less than $6 \mathrm{~mm}$ by a successful restoration of SR. Whereas, as for patients with severe leaflets tethering, the results favored surgical treatment over catheter ablation.

It is remarkable that not all patients with AFcaused moderate-severe TR would benefit from a rhythm control approach for treating AF. Patients with tethering height less than $6 \mathrm{~mm}$ who develop TRAF may represent a special category that derives significant clinical benefit from restoration of SR without surgery. This issue would be best answered in a prospective study.

TV surgery is the definitive therapy for most of the patients with severe symptomatic TR [22]. Most of the recommendations are targeted at patients who undergo concomitant left-sided heart procedures [23]. There is a paucity of recommendations regarding surgery in patients with TRAF. Due to lack of outcomes in literature, the current guidelines do not directly address the management of TRAF. TV annuloplasty is widely recommended for the treatment of functional TR [24]. The rationale for the currently used TV annuloplasty techniques is based primarily on the assumption that the major cause of functional TR is annular dilatation. However, recurrent $\geq 3+$ TR after repair occurs in $3-14 \%$ of patients with steady increase in its incidence over time and affects up to $20 \%$ of patients by 5 years [25, 26]. Severe TR with right-sided heart remodeling frequently presents with tethering of leaflets, which has been 


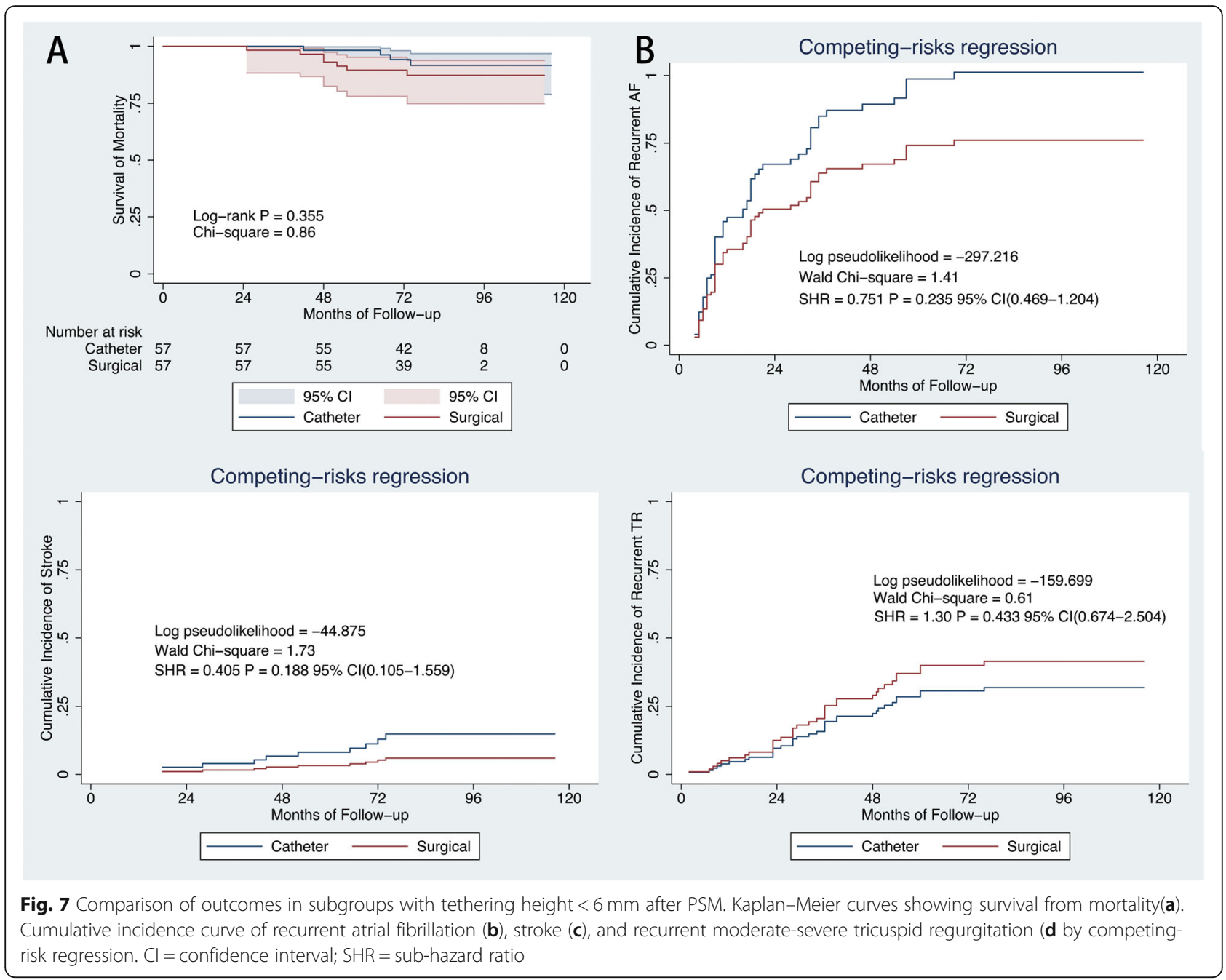

addressed as an independent predictive risk factor of recurrent TR [27, 28].

Therefore, simple annuloplasty techniques might not be adequate to correct TR in patients with severe tethering. Dreyfus first introduced a technique by using an autologous pericardial patch to enlarge the anterior tricuspid leaflet to overcome the tethering effects of the dilated RV [29]. It effectively increased the surface of coaptation by three-fold and allowed leaflet coaptation to take place within the RV at the level of tethered septal and posterior leaflets, while maintaining the leaflet mobility. This effectively compensated for severe leaflet tethering, as leaflet coaptation is achieved with reduced leaflet tension [30-32]. Recently, we have used an autologous pericardial patch to enlarge the anterior tricuspid leaflet in patients with tethering height more than $8 \mathrm{~mm}$. (Supplementary Figure 4) But the clinical results requires further investigation in patients with severe TR.

\section{Study limitations}

However, there are several limitations in this study that needs to be acknowledged. Firstly, the relatively limited sample size and inherent retrospective nature of the study inevitably led to missing data and recall bias. Differential losses to follow-up also biased this retrospective cohort study. Secondly, only 2dimensional echocardiographic parameters were used to evaluate RV geometry and TV tethering. Although 2D echocardiography is widely used in clinical practice and also for research currently, especially for patients with AF, 2D images lacked from inherent limitations such as incomplete comprehension of 3D tricuspid annulus. 3D echocardiography with one beat acquisition might provide more accurate measurements with more reproducible landmarks and could be used to confirm the present findings in further studies [33, 34]. Thirdly, in our subgroup analysis, although propensity-score matching adjusts for baseline 


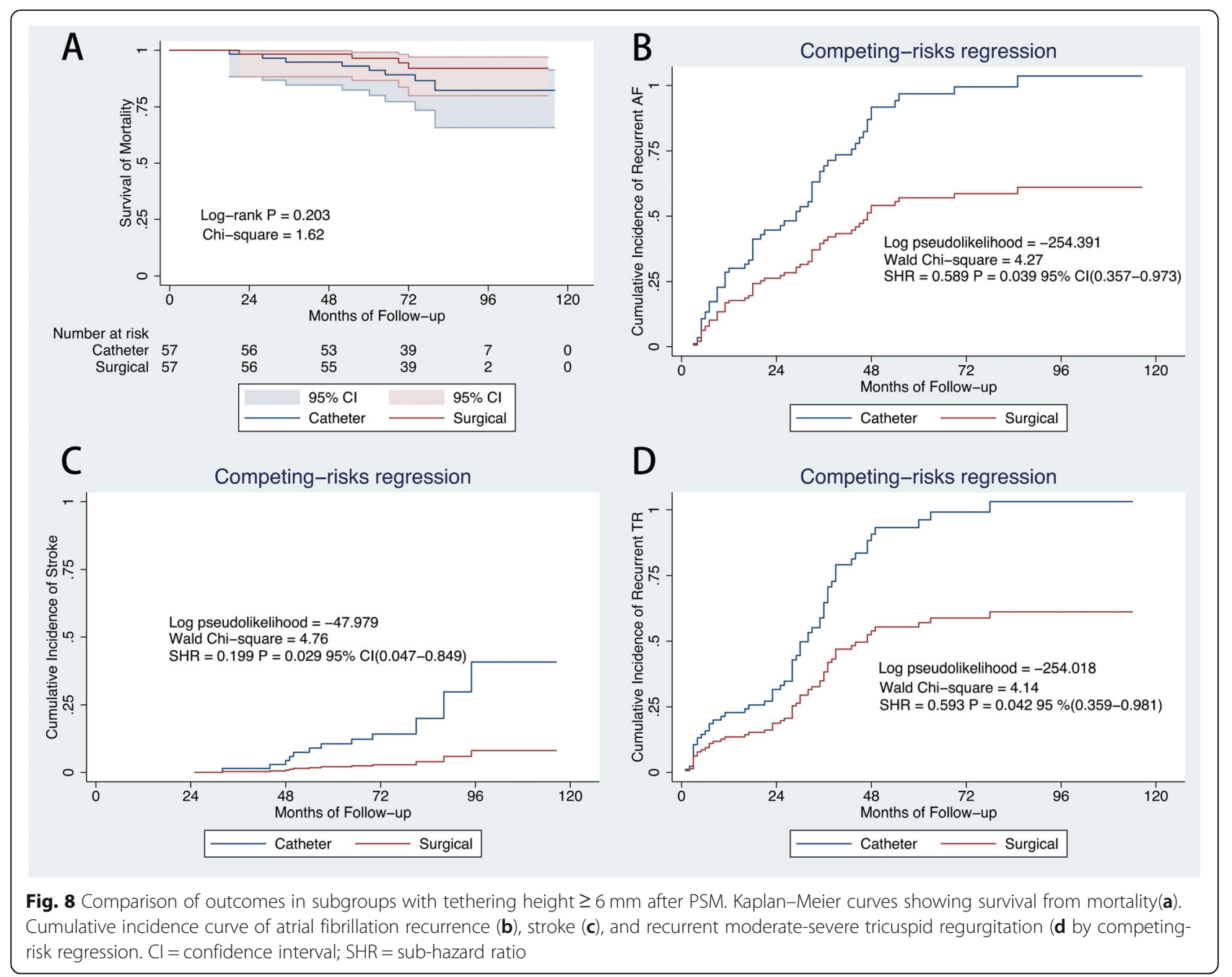

imbalances, the analysis does not control for unmeasured confounders. Moreover, small subgroup of patients and the analysis is likely underpowered, a longer-term follow-up results need be validated.

\section{Conclusions}

Our findings suggested that patients with TRAF, has right-sided heart dysfunction and less leaflets coaptation, which may be at risk of recurrence of AF and moderate-severe TR after catheter or surgical treatment. Despite having a moderate or greater degree of TR, patients with TRAF whose tethering height was less than $6 \mathrm{~mm}$ showed satisfied improvement in valve function with restoration of SR after catheter ablation. However, in patients with severe leaflets tethering, the results favored surgical treatment over catheter ablation. Since our study is retrospective in nature and whether all patients with TRAF should undergo above-mentioned strategy must be addressed in further prospective study.

\section{Supplementary information}

Supplementary information accompanies this paper at https://doi.org/10. 1186/s13019-020-01336-3.

\section{Additional file 1: Supplementary Table 1. Echocardiographic} characteristics of catheter cohort at baseline and follow-up. Supplementary Table 2. Echocardiographic characteristics of surgical cohort at baseline and follow-up. S Figure 1. Propensity-score matching for the total cohort. A, Dot plot of patients in either matched or unmatched groups. B, Histograms with overlaid kernel density estimates of standardized differences before and after matching. C, Distribution of propensity scores of surgical ("treated") and catheter cohort ("control") before and after matching with overlaid kernel density estimate. D, Line plot of standardized differences before and after matching. E, Dot plot of standardized mean differences (Cohen's d) for all covariates before and after matching. $\mathbf{S}$ Figure 2. Example of a patient in surgical cohort with a preoperational tethering height of $1.22 \mathrm{~cm}(\mathrm{~A})$, had a significant moderatesevere TR at 18 months follow-up with recurrent AF (B). $\mathbf{S}$ Figure 3. Example of a patient with a preoperational moderate-severe TR and 
tethering height of $0.4 \mathrm{~cm}$ in catheter cohort (A), had no TR at 24 months follow-up with sinus rhythm (B). S Figure 4. Example of using an autologous pericardial patch (white arrow) to enlarge the anterior tricuspid leaflet in patients with tethering height more than $8 \mathrm{~mm}$.

\section{Abbreviations}

AF: Atrial fibrillation; AUC: Area under the ROC curve; CHF: Congestive heart failure; Cls: Confidence intervals; PSM: Propensity-score match; PVAl: Pulmonary vein antrum isolation; RA: Atrial are; ROC: Receiver operating characteristic; RV: Right ventricular; RVFAC: Right ventricular fractional area change; RVSI: Right ventricular sphericity inde; SD: Standard deviation; SR: Sinus rhythm; TAD: Tricuspid annular dimension; TR: Tricuspid regurgitation; TV: Tricuspid valve; VC: Vena contracta

\section{Acknowledgements}

Not applicable.

\section{Authors' contributions}

Concept/design: J.W., S.L., J.D., C.M., X.M.; Data analysis/interpretation: J.W. S.L. J.H., Y.L.; Drafting article: J.W. S.L.; Critical revision of article: J.W.; Statistics: J.W.; Funding secured by J.W., Data collection, Q.Y., Y.Z., X.M. S.Z. K.L., M.H., W.Y., J.S... The author(s) read and approved the final manuscript.

\section{Funding}

This work was supported by the National Natural Science Foundation of China (Grant No. 88770320).

\section{Availability of data and materials}

The datasets used and/or analyzed during the current study are available from the corresponding author on reasonable request.

\section{Ethics approval and consent to participate}

The institutional review board at the Beijing Anzhen Hospital, Capital Medical University has approved the study.

\section{Consent for publication}

The need for patient consent was waived.

\section{Competing interests}

None.

\section{Author details}

'Department of Cardiac Surgery, Beijing Anzhen Hospital, Capital Medical University, No.2 Anzhen Road, Chaoyang District, Beijing 100029, PR China. 2Department of Cardiology, Beijing Anzhen Hospital, Capital Medical University, Beijing, China.

\section{Received: 3 April 2020 Accepted: 22 September 2020}

Published online: 29 September 2020

\section{References}

1. Zhao SX, Soltanzad N, Swaminathan A, Ogden WD, Schiller NB. Frequency and associated clinical features of functional tricuspid regurgitation in patients with chronic atrial fibrillation. Am J Cardiol. 2017;119:1371-7.

2. Dreyfus GD, Martin RP, Chan KMJ, Dulguerov F, Alexandrescu C. Functional tricuspid regurgitation: a need to revise our understanding. J Am Coll Cardiol. 2015;65:2331-6.

3. Prihadi EA, Delgado V, Leon MB, Enriquez-Sarano M, Topilsky Y, Bax J. Morphologic types of tricuspid regurgitation: characteristics and prognostic implications. JACC Cardiovasc Imaging. 2019;12:491-9.

4. Nishimura RA, Otto CM, Bonow RO, et al. 2017 AHA/ACC focused update of the 2014 AHA/ACC guideline for the Management of Patients with Valvular Heart Disease: a report of the American College of Cardiology/American Heart Association task force on clinical practice guidelines. Circulation. 2017; 135:e1159-95.

5. Dong J-Z, Sang C-H, Yu R-H, et al. Prospective randomized comparison between a fixed "2C3L" approach vs. stepwise approach for catheter ablation of persistent atrial fibrillation. Europace. 2016;17:1798-806.
6. Zhao $L$, Jiang $W$, Zhou $L$, et al. The role of valvular regurgitation in catheter ablation outcomes of patients with long-standing persistent atrial fibrillation. Europace. 2014;16:848-54.

7. Gertz ZM, Raina A, Saghy L, et al. Evidence of atrial functional mitral regurgitation due to atrial fibrillation. J Am Coll Cardiol. 2011;58:1474-81.

8. Ruaengsri C, Schill MR, Khiabani AJ, Schuessler RB, Melby SJ, Damiano RJ. The cox-maze IV procedure in its second decade: still the gold standard? Eur J Cardiothorac Surg. 2018;53:119-25.

9. Gillinov AM, Gelijns AC, Parides MK, et al. Surgical ablation of atrial fibrillation during mitral-valve surgery. N Engl J Med. 2015;372:1399-409.

10. Gelsomino S, La Meir M, Van Breugel HNAM, et al. Surgical ablation in patients undergoing mitral valve surgery: impact of lesion set and surgical techniques on long-term success. Europace. 2015;18:1528-37.

11. Badhwar V, Rankin JS, Damiano RJ, et al. The Society of Thoracic Surgeons 2017 clinical practice guidelines for the surgical treatment of atrial fibrillation. Ann Thorac Surg. 2017;103:329-41.

12. Wang J, Meng $X$, Li H, Cui Y, Han J, Xu C. Prospective randomized comparison of left atrial and biatrial radiofrequency ablation in the treatment of atrial fibrillation. Eur J Cardiothorac Surg. 2009;35:116-22.

13. Schiller NB, Shah PM, Crawford M, et al. Recommendations for quantitation of the left ventricle by two-dimensional echocardiography. American Society of Echocardiography Committee on standards, subcommittee on quantitation of two-dimensional echocardiograms. J Am Soc Echocardiogr. 1989;2:358-67.

14. Miyatake $\mathrm{K}$, Okamoto $\mathrm{M}$, Kinoshita $\mathrm{N}$, et al. Evaluation of tricuspid regurgitation by pulsed Doppler and two-dimensional echocardiography. Circulation. 1982;66:777-84.

15. Kaul S, Tei C, Hopkins JM, Shah PM. Assessment of right ventricular function using two-dimensional echocardiography. Am Heart J. 1984; 107:526-31.

16. Matsunaga A, Duran CMG. Progression of tricuspid regurgitation after repaired functional ischemic mitral regurgitation. Circulation. 2005;112:1453-7.

17. Gould L, Weber D, Schaffer Al, O'Connor RA. Does atrial fibrillation lead to tricuspid insufficiency? Am J Cardiol. 1965:16:188-94.

18. Mangieri A, Montalto C, Pagnesi M, et al. Mechanism and implications of the tricuspid regurgitation: from the pathophysiology to the current and future therapeutic options. Circ Cardiovasc Interv. 2017;10:1514.

19. Park J-H, Shin S-H, Lee $\mathrm{M}-\mathrm{J}$, et al. Clinical and echocardiographic factors affecting tricuspid regurgitation severity in the patients with lone atrial fibrillation. J Cardiovasc Ultrasound. 2015;23:136-7.

20. Chou S-H, Kuo C-T, Hsu L-A, Ho W-J, Wang C-L. Single-beat determination of right ventricular function in patients with atrial fibrillation. Echocardiography. 2010;27:1188-93.

21. Celebi $\mathrm{O}$, Agactigen $\mathrm{A}$, Sahin T, et al. Right ventricular functions in patients with permanent and paroxysmal atrial fibrillation. Eur J Echocardiogr. 2005;6: S24.

22. Zack CJ, Fender EA, Chandrashekar $P$, et al. National Trends and outcomes in isolated tricuspid valve surgery. J Am Coll Cardiol. 2017;70: 2953-60.

23. King RM, Schaff HV, Danielson GK, et al. Surgery for tricuspid regurgitation late after mitral valve replacement. Circulation. 1984;70:1193-7.

24. Rogers $J H$, Rogers JH, Bolling SF, Bolling SF. Surgical approach to functional tricuspid regurgitation: should we be more aggressive? Curr Opin Cardiol. 2014;29:133-9.

25. McCarthy PM, Bhudia SK, Rajeswaran J, et al. Tricuspid valve repair: durability and risk factors for failure. J Thorac Cardiovasc Surg. 2004;127: 674-85.

26. Marquis-Gravel G, Bouchard D, Perrault LP, et al. Retrospective cohort analysis of 926 tricuspid valve surgeries: clinical and hemodynamic outcomes with propensity score analysis. Am Heart J. 2012;163:851-858. e1.

27. Fukuda S. Determinants of Recurrent or Residual Functional Tricuspid Regurgitation After Tricuspid Annuloplasty. Circulation. 2006;114:1582-7.

28. Fukuda S, Gillinov AM, McCarthy PM, Matsumura Y, Thomas JD, Shiota T. Echocardiographic follow-up of tricuspid Annuloplasty with a new threedimensional ring in patients with functional tricuspid regurgitation. J Am Soc Echocardiogr. 2007;20:1236-42.

29. Dreyfus GD, Raja SG, John Chan KM. Tricuspid leaflet augmentation to address severe tethering in functional tricuspid regurgitation. Eur J Cardiothorac Surg. 2008;34:908-10. 
30. Choi JB, Kim KH, Kim MH. A modified tricuspid ring Annuloplasty to reduce residual regurgitation in functional tricuspid regurgitation. J Card Surg. 2010; 25:647-50.

31. Quarti A, lezzi F, Soura E, Colaneri M, Pozzi M. Anterior and posterior leaflets augmentation to treat tricuspid valve regurgitation. J Card Surg. 2015;30:421-3.

32. Pettinari $M$, Bertrand $P$, Van Kerrebroeck $C$, Vandervoort $P$, Gutermann $H$, Dion R. Mid-term results of leaflet augmentation in severe tricuspid functional tethering. Eur J Cardiothorac Surg. 2016;50:504-8.

33. Utsunomiya $\mathrm{H}$, Itabashi $\mathrm{Y}$, Mihara $\mathrm{H}$, et al. Functional tricuspid regurgitation caused by chronic atrial fibrillation: a real-time 3-dimensional transesophageal echocardiography study. Circ Cardiovasc Imaging. 2017;10: e004897.

34. Muraru D, Hahn RT, Soliman Ol, Faletra FF, Basso C, Badano LP. 3dimensional echocardiography in imaging the tricuspid valve. JACC Cardiovasc Imaging. 2019;12:500-15.

\section{Publisher's Note}

Springer Nature remains neutral with regard to jurisdictional claims in published maps and institutional affiliations.

Ready to submit your research? Choose BMC and benefit from:

- fast, convenient online submission

- thorough peer review by experienced researchers in your field

- rapid publication on acceptance

- support for research data, including large and complex data types

- gold Open Access which fosters wider collaboration and increased citations

- maximum visibility for your research: over $100 \mathrm{M}$ website views per year

At $\mathrm{BMC}$, research is always in progress.

Learn more biomedcentral.com/submissions 